\title{
Quality Assessment and Antioxidant Activities of the Blossoms of Inula Nervosa Wall
}

\author{
Li-Xia Hu, ${ }^{1}$ Mei-Feng Luo, ${ }^{2}$ Wen-Jing Guo, ${ }^{3}$ Xiao He, ${ }^{3}$ Jun Zhou, ${ }^{1}$ Xiao-Yu Qiu, ${ }^{3}$ \\ Jian-Ping Gong, ${ }^{3, *}$ Meng-Chu Li, ${ }^{4}$ Xin-Tao Chen, ${ }^{2}$ Dong Wu, ${ }^{4}$ and \\ Wen-Ping Huang (1) ${ }^{4, *}$
}

${ }^{1}$ Jiangxi Chest Hospital, 346 Dieshan Road, Nanchang, 330006 Jiangxi, China, ${ }^{2}$ The Third Affiliated Hospital of Nanchang University, 128 Xianshang North Road, Nanchang, 330006 Jiangxi, China, ${ }^{3}$ Jiangxi University of Traditional Chinese Medicine, 1688 Meiling Avenue, Nanchang, 330004 Jiangxi, China, ${ }^{4}$ The National Pharmaceutical Engineering Center (NPEC) for Solid Preparation in Chinese Herbal Medicine, Jiangxi University of Traditional Chinese Medicine, 56 Yangming Road, Nanchang, 330006 Jiangxi, China

*Corresponding author’s e-mail: 1815784092@qq.com and huangwenping89@126.com

\begin{abstract}
Background: Currently, although Inula nervosa Wall is substantially investigated, little is understood about blossoms of Inula nervosa Wall (BINW).

Objective: In this work, we systematically investigated the antioxidant activity of the extract from BINW by various standard assays including 1,1-diphenyl-2-picrylhydrazyl (DPPH) free radical ability, 2,2' -azino-bis (3-ethylbenzothiazoline-6-sulfonic acid) di-ammonium salt radical cation (ABTS), and ferric reducing antioxidant potential (FRAP).

Methods: Chemical compounds were tentatively identified through an UHPLC-QTOF-MS system. Furthermore, the contents of nine compounds were detected with UHPLC method coupled with photodiode array (PDA) detector. By carefully analyzing the quantitative data via clusters analysis and principal component analysis (PCA).

Results: Forty-six compounds were tentatively identified, and our results showed that nine compound samples in 21 batches of BINW collected from different areas could be differentiated and analyzed by a heatmap visualization. In addition, the contents of nine compounds (flavonoids, phenolic acids) exhibited a total of higher amounts and better antioxidant activities from Yunnan than those from the other three origins.

Conclusions: Our study not only developed a powerful platform to explain the difference between traditional Chinese medicines species that are closely related through the chemometric and chemical profiling, but also presented a useful method to establish quality criteria of BINW with multiple origins.

Highlights: To characterize the BINW in detail, we not only performed DPPH, FRAP, and ABTS assays to investigate its antioxidant activity, but also established UHPLC-QTOF-MS/MS- and UHPLC-PDA-based methods to comprehensively identify and qualitatively analyze its components.
\end{abstract}

An imbalance of the homeostasis between pro-oxidant and antioxidant could induce oxidative stress and generate toxic radical species. Varieties of oxidative stress in most aerobic organisms can have many effects on different 
biomacromolecules such as including DNA, proteins, and membrane lipids, which would induce various chronic disorders such as cancer, aging, inflammation, type 2 diabetes, and neurodegenerative diseases (1-5). Reactive oxygen species at low levels are essential for life, however excessive amounts could have a negative impact on human health. Hence, study of antioxidants of greater efficacy and lower toxicity was worthy of considerable attention. To evaluate antioxidant effects, there are many efforts spent in establishing relevant detection methods, among which 2,2'-azino-bis (3-ethylbenzothiazoline-6-sulfonic acid) diammonium salt radical cation (ABTS), ferric reducing antioxidant potential (FRAP), and 1,1-diphenyl2-picrylhydrazyl (DPPH), reducing power assays, were frequently employed (6-9).

Inula nervosa Wall (INW), commonly called "Xiaoheiyao" in China, is currently being extensively used as an expectorant and digestant in Chinese medicine. In addition, blossoms of INW (BINW) have been utilized as a popular kind of wine used by the Tong people, one of the minorities in China (10). Recently, reports indicated that many active compounds from INW, such as polysaccharides, phenolic acids, and flavonoids (11-13), exhibit numerous beneficial effects including antiinflammatory, neuroprotective, antioxidant, hepatoprotective, and anti-cancer assistance (14-18). However, little is understood about the antioxidant effects from extracts of BINW and their bioactive components. Analytical techniques such as LC-MS, HPLC, and GC are the fundamental ways to analyze components in vivo and in vitro (19-24). Up until now, a series of analytical methods such as LC-MS, HPLC-PDA, and GC-MS has been used for qualitative and quantitative analysis of INW $(25,26)$. However, there were few compounds quantified because of the lack of good standards. To this end, this study will establish a series of good standards, aimed at quality assessment and antioxidant activities for these samples from different areas of BINW. First, an UHPLC-QTOF-MS/MS method is established to identify chemical constituents. Next, 21 batches of samples from different areas of BINW were used to determine the contents of multiple compounds and also to test the antioxidant capacities through DPPH, ABTS, and FRAP assay.

\section{Materials and Methods}

\section{Chemicals and Reagents}

The nine standard chemical compounds including kaempferol, luteolin, kaempferol-3-O-rutinoside, nepetin, 3,5-dicaffeoylquinic acid, chlorogenic acid, quercetin, 1,5-DQA: 1,5-dicaffeoylquinic acid, and thymol were bought from Chengdu DeSiTe Biotechnology Co., Ltd. (Chengdu, China). All compounds' purity was higher than $98 \%$ which was detected through HPLC-(PDA) detector analysis. HPLC-grade formic acid, $\mathrm{MeOH}$, and EtOH were bought from Thermo Fisher Scientific (China) Co., Ltd. Distilled water was bought from Watsons Water (Hong Kong, China). DPPH, ABTS, FRAP, and Vitamin $C$ were bought from Beijing Solarbio Science \& Technology Co., Ltd.

\section{Plant Materials and Sample Preparation}

The samples of BINW, totaling 21 batches in all, were gathered from the Hebei, Shandong, Yunnan, and Hubei provinces and verified by Professor Jianping Gong from Jiangxi University of Traditional Chinese Medicine. Detailed information on the samples are shown in Supplemental Table S1.
Extraction of each dried sample was obtained from $0.5 \mathrm{~g}$ powder dissolved in a $50 \mathrm{~mL}$ solution $\left(\mathrm{CH}_{3} \mathrm{OH}: \mathrm{H}_{2} \mathrm{O}=7: 3\right)$. Later, the solution was refluxed for $30 \mathrm{~min}$, and then centrifuged at 13 $\mathrm{krpm}$ for $10 \mathrm{~min}$, which were finally used for further analyze.

A stock solution of nine standards was prepared by dissolving accurately weighed standards in methanol to make the concentrations of 184.0, 123.3, 645.5, 38.0, 36.3, 19.0, 62.0, 22.0, and $520.0 \mu \mathrm{g} / \mathrm{mL}$ for chlorogenic acid (C1), 3,5-dicaffeoylquinic acid (C2), 1,5-dicaffeoylquinic acid (C3), kaempferol-3-O-rutinoside (C4), quercetin (C5), luteolin (C6), nepetin (C7), kaempferol (C8), and thymol (C9), respectively. The stock solution was then diluted to a series of working solutions in six levels by methanol solution, and all solutions were kept at $4^{\circ} \mathrm{C}$.

\section{Antioxidant Activities}

All samples were diluted with methanol and then a series of extract concentrations were prepared. In addition, the antioxidant activities were tested via the methods of DPPH, FRAP, and ABTS.

\section{DPPH Assay}

DPPH activity assay was determined based on a previous report (27). Briefly, the solution of extracts $(100 \mu \mathrm{L})$ were mixed with $\mathrm{DPPH}(100 \mu \mathrm{L})$ and the solutions were put it in the dark for $30 \mathrm{~min}$ to prepare a stock solution. Finally, the $\mathrm{IC}_{50}$ values were calculated based on the absorbance characterized at $517 \mathrm{~nm}$.

\section{FRAP Assay}

Ferric activity assay was determined based on the Benzie and Strain method with minor modifications (28). Samples $(5 \mathrm{mg} /$ $\mathrm{mL}$ ) were dissolved in a $1 \mathrm{~mL}$ solution containing potassium ferricyanide $(1 \%)$ and phosphate buffer $(0.2 \mathrm{M}, \mathrm{pH}=6.6)$, following incubation at $50^{\circ} \mathrm{C}$ for $30 \mathrm{~min}$. The mixed sample $(1 \mathrm{~mL})$ was then added to a stock solution including deionized water $(1 \mathrm{~mL})$, $10 \%$ trichloroacetic acid $(1 \mathrm{~mL})$, and $0.1 \% \mathrm{FeCl}_{3}(1 \mathrm{~mL})$. Finally, the $\mathrm{IC}_{50}$ values calculated through absorbance were measured at $700 \mathrm{~nm}$ after depositing the solution in the dark for $10 \mathrm{~min}$.

\section{ABTS Assay}

An ABTS solution was prepared by the traditional method. Extracts $(100 \mu \mathrm{L})$ were mixed with the ABTS solution $(100 \mu \mathrm{L})$ and put it in the dark for $30 \mathrm{~min}$. The $\mathrm{IC}_{50}$ values calculated through absorbance measured $734 \mathrm{~nm}$ (29).

\section{The Analytical Condition of the UHPLC-QTOF-MS/MS Experiments}

The UHPLC analysis for BINW was performed with an LC-30 type instrument system from Shimadzu (Kyoto, Japan). The separation was carried out using a Welch HPLC column (Ultimate $\mathrm{XB} \mathrm{C} 18: 100 \mathrm{~mm} \times 2.1 \mathrm{~mm}, 1.7 \mu \mathrm{m}$ ) and the flow rate was fixed at $0.25 \mathrm{~mL} \mathrm{per} / \mathrm{min}$. The temperature of the column was kept at $35^{\circ} \mathrm{C}$, and the volume of the sample injected was $2 \mu \mathrm{L}$. The gradient elution system was established by $\mathrm{CH}_{3} \mathrm{CN}$ (A solvent) and $0.1 \%$ aqueous formic acid (B solvent): $98 \%$ B (0-4 min), 98-95\% B (4-6 $\mathrm{min}), 95-90 \%$ B (6-10 $\mathrm{min}), 90-82 \%$ B (10-22 $\mathrm{min}), 82-75 \%$ B (22-32 min), $75-30 \%$ B (32-42 min), 30-98\% B (42-52 min), 98\% B (52-55 min).

The UHPLC-QTOF-MS/MS was used by an AB SCIEX Triple $\mathrm{TOF}^{\mathrm{TM}} 5600$ + system (Boston, CA, USA). The optimized MS condition was listed as follows: decluttering potential (DP) and the ion spray voltage were fixed at \pm 100 and $\pm 4500 \mathrm{~V}$, respectively. 
The temperature for ion source was set at $550^{\circ} \mathrm{C}$; curtain gas, nebulizer gas (Gas 1) and heater gas (Gas 2) were set at 50, 50, and 25 psi, respectively. The collision energy (CE) was fixed at 40 and $-35 \mathrm{eV}$ for BINW, respectively.

\section{UHPLC Chromatographic Conditions}

The UHPLC system (Shimadzu, Kyoto, Japan) consisting of an LC-300type instrument system was applied to quantitative determination for different compounds. The separation experiment was carried out via an Agilent column $(100 \mathrm{~mm} \times 3.0 \mathrm{~mm}$, $2.7 \mu \mathrm{m}$, Poroshell $120 \mathrm{C}_{18}$ ). The flow rate for the system was set at $0.30 \mathrm{~mL} / \mathrm{min}$ with the temperature fixed at $35^{\circ} \mathrm{C}$. The wavelength for the detection wavelength was set at $254 \mathrm{~nm}$, and the sample volume for the injection was $5 \mu \mathrm{L}$. The mobile phase included $\mathrm{CH}_{3} \mathrm{CN}$ (A solvent) and $0.1 \% \mathrm{CH}_{3} \mathrm{COOH}$ (B solvent), which were used as a gradient elution system with an equilibration time of $15 \mathrm{~min}$, the details of which are shown below: $98-98 \%$ B (0-4 min), 98-95\% B (4-6 min), 95-90\% B (6-10 min), 90-80\% B (10-20 min), 80-73\% B (20-30 min), 73-75\% B (30-37 min), 75-68\% B (37-45 min), 68-42\% B (45-68 min), 42-75\% B (68-75 min), $75-98 \%$ B (75-82 $\mathrm{min})$, and $98-98 \%$ B (82-90 $\mathrm{min})$. The acceptable ranges of the linearity, detection, and quantification limit of precision, repeatability, stability, and accuracy were followed according to Pharmacopoeia guidelines.

\section{Results and Discussion}

\section{Antioxidant Assay for the Extracts of BINW}

The human body contains a variety of free radical, light radicals, superoxide anions, and peroxide radicals ozone, as well as various antioxidants. However, due to their diverse natures, a simplex antioxidant may exhibit different effects when suffering various free radicals. Therefore, diverse methods for antioxidant evaluation should be applied in assessing the capability of total antioxidants in substances comprehensively. Based on these reasons, the antioxidant activity of BINW was evaluated by using the FRAP, DPPH, and ABTS methods.

DPPH is an effective method for testing the antioxidant abilities of BINW. Based on the results obtained, the extracts of BINW from different areas also show different free radical scavenging abilities. The $\mathrm{IC}_{50}$ values were: Yunnan samples $1.40 \pm 0.03 \mathrm{mg} / \mathrm{mL} \sim 1.57 \pm 0.01 \mathrm{mg} / \mathrm{mL} ; \quad$ Shandong samples $4.70 \pm 0.04 \mathrm{mg} / \mathrm{mL} \sim 6.05 \pm 0.00 \mathrm{mg} / \mathrm{mL} ;$ Hebei samples $2.83 \pm$ $0.01 \mathrm{mg} / \mathrm{mL} \sim 3.18 \pm 0.03 \mathrm{mg} / \mathrm{mL} ;$ and Hubei samples $3.63 \pm$ $0.03 \mathrm{mg} / \mathrm{mL} \sim 4.01 \pm 0.01 \mathrm{mg} / \mathrm{mL}$. The tendency of $\mathrm{IC}_{50}$ values calculated from ABTS and FRAP was similar to those from DPPH shown in Table 1. In short, it was clearly shown that the samples from Yunnan overall have better antioxidant activity than those from the other three areas.

\section{Optimization of Extraction and Analysis Conditions}

Different extraction solvents $(95,75,50$, and $25 \%$ methanol or ethanol) and extraction time (60 or $30 \mathrm{~min}$ ) were applied to find the most efficient extraction method. The optimized method was reflux extraction by $70 \%$ methanol at room temperature for $30 \mathrm{~min}$. The extracts of BINW were tested and evaluated in two ionization modes (positive and negative) by UHPLC-Q-TOF-MS/ MS. In addition, several experiments on mobile phase combinations such as $0.1 \%$ formic acid with acetonitrile or methanol were conducted. The results indicated that $\mathrm{CH}_{3} \mathrm{CN}$ containing formic acid $(0.1 \%, v / v)$ showed better sufficient ionization and separation.
Table 1. Antioxidant activity of blossoms of Inula nervosa Wall [IC value $(\mathrm{mg} / \mathrm{mL})]^{\mathrm{a}}$

\begin{tabular}{lccc}
\hline Sample source & DPPH & FRAP & ABTS \\
\hline Shandong, S1 & $4.70 \pm 0.04$ & $7.56 \pm 0.02$ & $19.90 \pm 0.05$ \\
Shandong, S2 & $5.04 \pm 0.02$ & $6.56 \pm 0.02$ & $20.36 \pm 0.08$ \\
Shandong, S3 & $4.89 \pm 0.02$ & $7.12 \pm 0.03$ & $21.15 \pm 0.02$ \\
Shandong, S4 & $6.05 \pm 0.00$ & $7.50 \pm 0.02$ & $20.36 \pm 0.06$ \\
Yunnan, S5 & $1.57 \pm 0.01$ & $1.34 \pm 0.02$ & $6.18 \pm 0.03$ \\
Yunnan, S6 & $1.44 \pm 0.02$ & $1.16 \pm 0.02$ & $5.64 \pm 0.03$ \\
Yunnan, S7 & $1.42 \pm 0.02$ & $1.41 \pm 0.01$ & $7.95 \pm 0.03$ \\
Yunnan, S8 & $1.40 \pm 0.03$ & $1.43 \pm 0.05$ & $8.50 \pm 0.07$ \\
Hebei, S9 & $2.96 \pm 0.02$ & $2.54 \pm 0.04$ & $11.40 \pm 0.03$ \\
Hebei, S10 & $2.83 \pm 0.01$ & $2.85 \pm 0.03$ & $13.01 \pm 0.06$ \\
Hebei, S11 & $2.94 \pm 0.03$ & $2.97 \pm 0.07$ & $13.07 \pm 0.03$ \\
Hebei, S12 & $2.89 \pm 0.02$ & $3.08 \pm 0.01$ & $13.66 \pm 0.07$ \\
Hebei, S13 & $3.16 \pm 0.07$ & $3.18 \pm 0.07$ & $14.87 \pm 0.05$ \\
Hebei, S14 & $3.18 \pm 0.03$ & $3.16 \pm 0.00$ & $14.88 \pm 0.02$ \\
Hebei, S15 & $3.14 \pm 0.04$ & $3.22 \pm 0.00$ & $13.14 \pm 0.07$ \\
Hubei, S16 & $3.85 \pm 0.02$ & $4.13 \pm 0.02$ & $16.04 \pm 0.02$ \\
Hubei, S17 & $3.63 \pm 0.03$ & $4.29 \pm 0.04$ & $16.06 \pm 0.05$ \\
Hubei, S18 & $3.96 \pm 0.07$ & $4.43 \pm 0.07$ & $17.00 \pm 0.03$ \\
Hubei, S19 & $3.77 \pm 0.05$ & $4.84 \pm 0.05$ & $17.17 \pm 0.07$ \\
Hubei, S20 & $3.95 \pm 0.04$ & $4.86 \pm 0.02$ & $17.62 \pm 0.03$ \\
Hubei, S21 & $4.01 \pm 0.01$ & $5.08 \pm 0.03$ & $18.92 \pm 0.06$ \\
Vitamin C & $0.12 \pm 0.01$ & $0.32 \pm 0.01$ & $1.23 \pm 0.02$ \\
\hline
\end{tabular}

${ }^{a}$ Each value in the table is presented as the mean $\pm \operatorname{SD}(n=3)$.

\section{Identification and Characterization of the Compounds from BINW by the Combined Instrument System of UHPLC-QTOF-MS/MS}

The basic peak chromatograms (BPC) of BINW in two ionization modes (positive and negative) are shown in Figure 1. Forty-six compounds were discovered and, preliminarily, evaluated, including 10 flavonoids, 8 phenolic acids, and another 28 compounds (including coumarins, anthraquinones, glycosides, etc.) (30-53). The retention time and fragment information were compared with the reference standard, and 11 compounds were successfully identified and confirmed by analyzing the mass results. The detailed MS data of identified compounds is listed in Table 2. For instance, there is a molecular ion $[M-H] m / z$ 179.0347 for peak 12 , and it split into an ion at $\mathrm{m} / \mathrm{z} 161.0380$ $(\triangle \mathrm{m}=18)$ due to the splitting of $\mathrm{H}_{2} \mathrm{O}$. The fragment of $\mathrm{m} / \mathrm{z}$ 135.0456, and 107.0511 were due to the loss of $\mathrm{CO}_{2}$ and $\mathrm{CO}$. Furthermore, taking peak 15 as another example, there is a protonated molecular ion $[\mathrm{MH}]$ at $\mathrm{m} / \mathrm{z}$ 609.1854. Due to the cleavage of glucose bond, it disintegrates to ion at $\mathrm{m} / \mathrm{z} 447.0970$ $(\triangle M=162)$. The fragments of $m / z 285.0411$ and 257.0275 were due to the loss of glucose and CO.

\section{UHPLC-PDA Method Validation}

The chromatogram of nine standard compound mixtures are shown in Figure 2A and a sample chromatogram is shown in Figure 2B. We first confirmed that all calibration curves exhibited good linearity within the test ranges of our test experiment. Then the LOD was determined in the ranges of $0.23-10.24 \mu \mathrm{g} / \mathrm{mL}$ as the LOQs were $0.77-34.13 \mu \mathrm{g} / \mathrm{mL}$ (Supplemental Table S2). The RSD data was less than $1.00 \%$ in terms of both intra- and interday precisions. Stability was analyzed in $24 \mathrm{~h}$ and the RSD results were in the range of $0.11-1.05 \%$. Repeatability was evaluated and performed with 6 individual samples each (S13, Hebei) and RSD values did not reach $2.00 \%$. The average recoveries 

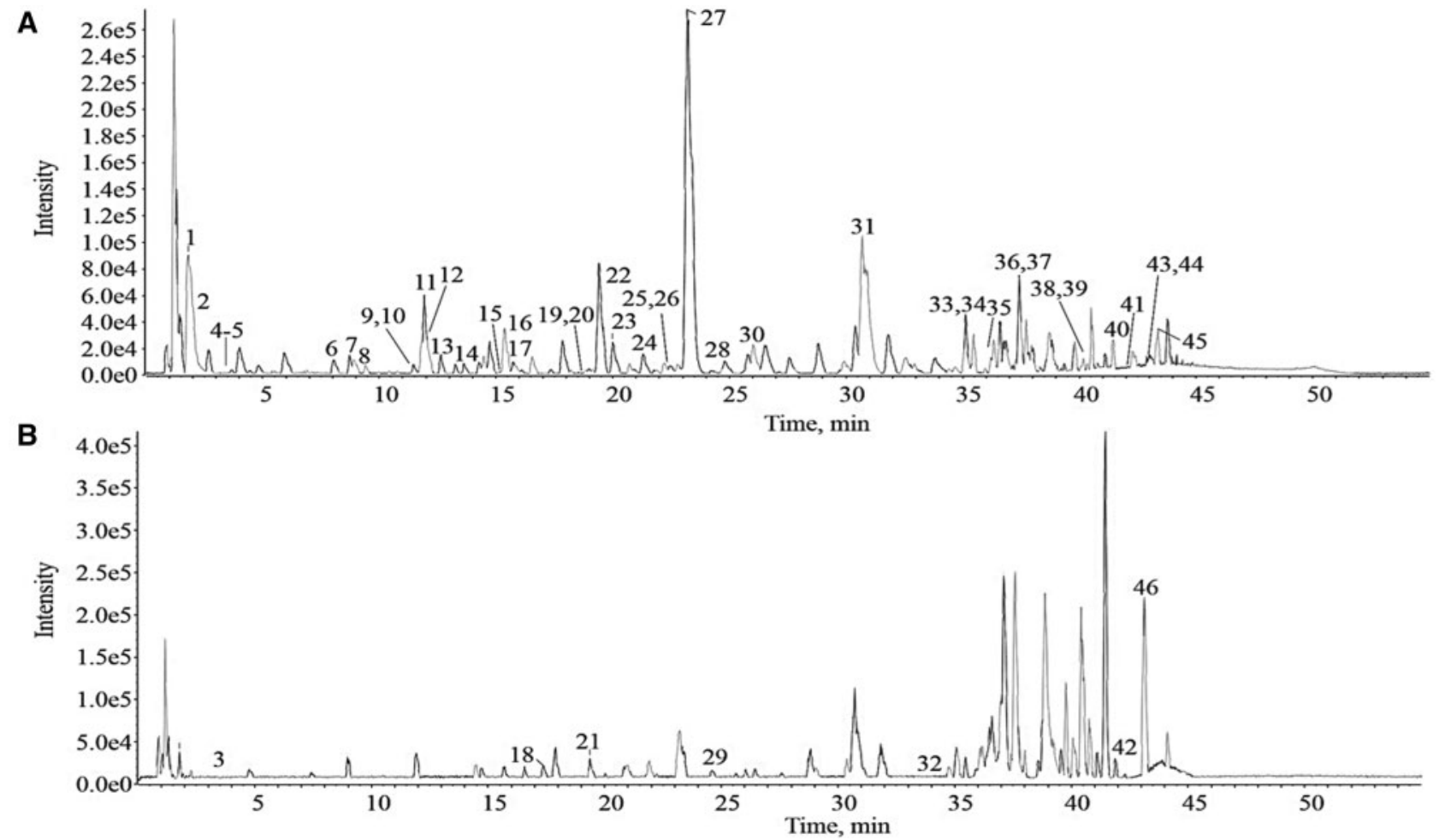

Figure 1. The basic peak chromatogram (BPC) of BINW in the negative (A) and positive (B) ion modes.
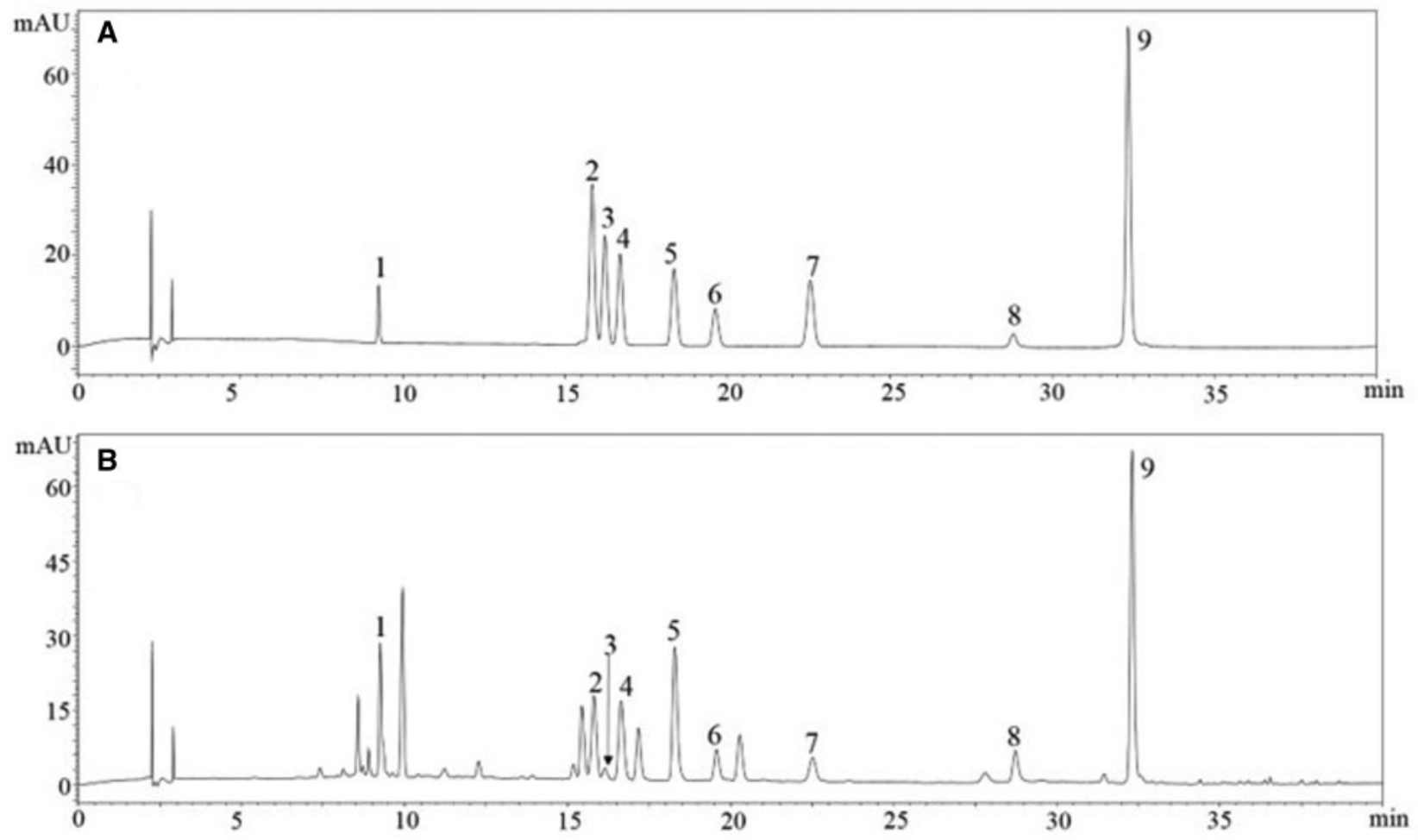

Figure 2. (A) Chromatogram of nine mixture standard compounds: Chlorogenic acid (C1), 3,5-Dicaffeoylquinic acid (C2), 1,5-Dicaffeoylquinic acid (C3), Kaempferol-3-Orutinoside (C4), Quercetin (C5), Luteolin (C6), Nepetin (C7), Kaempferol (C8), and Thymol (C9). (B) Sample chromatogram. 


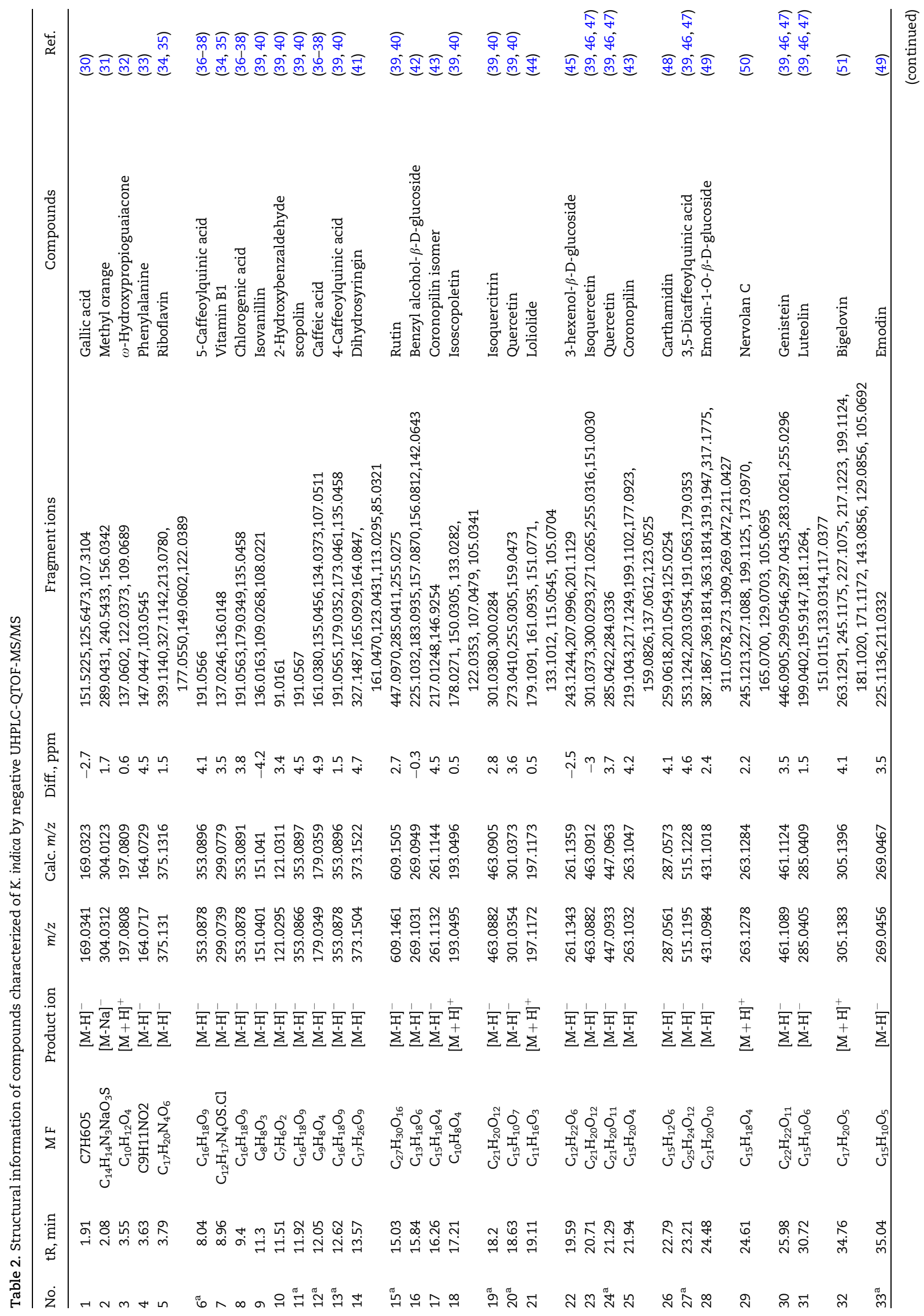




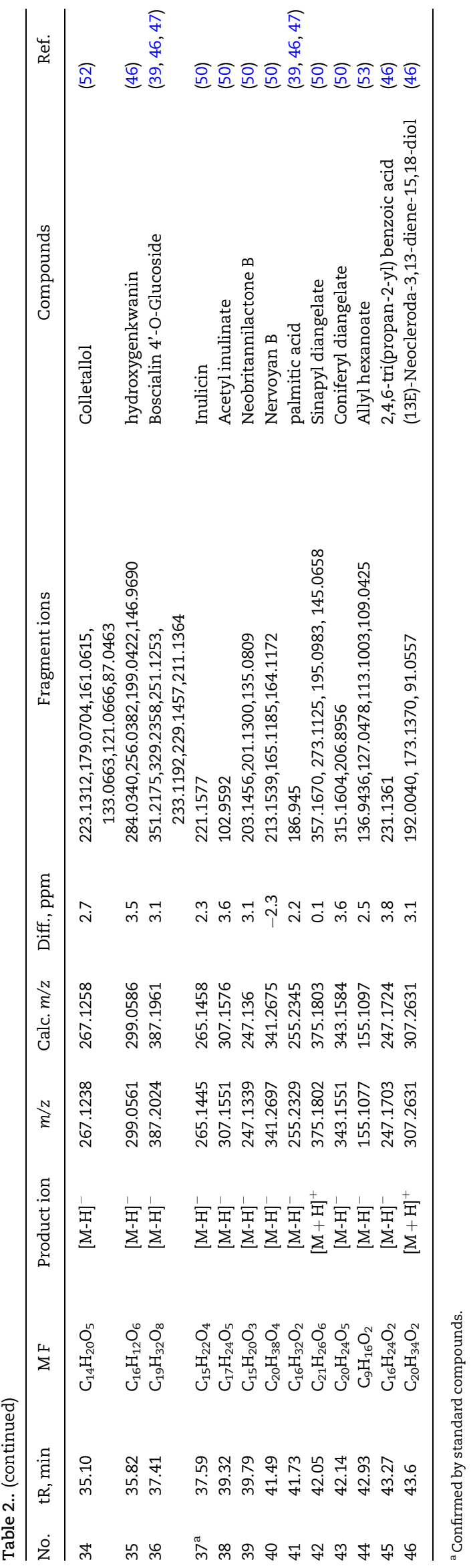

were in the range of $96.05-103.01 \%$ while the RSD data was determined in the range of $0.45-2.74 \%$. Our results showed that the analytical method established here is successfully used to quantify the multi-compounds in BINW (Supplemental Table S3).

\section{Quantitative Analysis of the Nine Compounds in BINW}

The contents of the nine compounds were tested in 21 BINW samples. As shown in Table 3, it can clearly be seen that the total contents of nine monomers in Yunnan province were the highest among these batches of samples from different origins (Figure 3). In addition, our results also suggested that 1,5-dicaffeoylquinic acid (C3) and thymol (C9) may be the basic and key constituents in BINW.

\section{Discrimination of the BINW from Different Areas based on the Chemometrics}

The quantitative data was analyzed both by IBM SPSS statistics and SIMCA-P 12.0 software to discriminate BINW samples from different areas. With the cluster analysis by SPSS, all data can be divided into four categories that corresponded to four different origins (Figure 4). Principal component analysis (PCA) was utilized to interpret the trend of the classification. The PCA scores plot (Figure 5) demonstrated a visible separation combined two constituents occupying $82.72 \%$ of the variance in total among 21 samples. Therefore, these data clearly demonstrated that chemometrics software was a useful instrument to classify the areas of BINW.

\section{Distribution of the Nine Compounds in BINW from Different Areas}

Although a clear discrimination of 4 groups of BINW samples was successfully confirmed and investigated, the distribution of the 9 compounds in 21 BINW samples was still unknown. Therefore, after Pareto calibration, the mean centered method was used to analyze the visualization of thermogram for unsupervised clustering, and the situation of these nine monomers can be clearly understood. As shown in Figure 6, BINW samples were obviously discriminated by hierarchical clustering analysis (HCA). The distribution of nine compounds in the four groups of BINW samples are deserving of notice. The compounds of kaempferol-3-O-rutinoside (C4), quercetin (C5), and luteolin (C7) could be marked to distinguish Yunnan samples from the other three area samples. Otherwise, chlorogenic acid (C1) and 3,5dicaffeoylquinic acid (C2) could be taken into consideration in Shandong samples for the difference between the other three areas samples. Due to the variation of findings in the chemical constituents from the different areas samples, our results could explain the significant discrimination among these samples from Yunnan, Shandong, Hebei, and Hubei, thus probably influencing their pharmacological activities which could be further determined in the future.

\section{Conclusions}

In this paper, in an effort characterize BINW in detail, we not only performed DPPH, FRAP, and ABTS assays not only to investigate antioxidant activity but also to establish UHPLC-QTOFMS/MS- and UHPLC-PDA-based methods to comprehensively identify and qualitatively analyze their components. Interestingly, our results showed that 46 compounds in total, 
Table 3. The contents (mg/g) of the 9 marker compounds in 21 samples of BINW

\begin{tabular}{|c|c|c|c|c|c|c|c|c|c|c|c|}
\hline Sample & Location & C1 & C2 & C3 & C4 & C5 & C6 & C7 & $\mathrm{C} 8$ & $\mathrm{C9}$ & Total contents \\
\hline $\mathrm{S} 1$ & Shandong & $1.46^{\mathrm{a}}$ & 1.33 & 7.07 & 0.36 & 0.48 & 0.16 & 0.88 & 0.16 & 5.54 & 17.44 \\
\hline $\mathrm{S} 2$ & Shandong & 1.42 & 1.15 & 6.93 & 0.36 & 0.40 & 0.17 & 0.90 & 0.16 & 5.64 & 17.13 \\
\hline S3 & Shandong & 1.41 & 1.40 & 7.24 & 0.35 & 0.43 & 0.25 & 0.93 & 0.15 & 5.51 & 17.67 \\
\hline S4 & Shandong & 1.39 & 1.42 & 7.49 & 0.36 & 0.49 & 0.28 & 0.91 & 0.16 & 6.09 & 18.58 \\
\hline S5 & Yunnan & 5.70 & 7.25 & 19.89 & 1.43 & 1.49 & 0.77 & 1.49 & 0.38 & 16.11 & 54.50 \\
\hline S6 & Yunnan & 5.03 & 6.95 & 20.35 & 1.45 & 1.51 & 0.77 & 1.48 & 0.38 & 15.43 & 53.35 \\
\hline S7 & Yunnan & 5.28 & 7.11 & 20.34 & 1.48 & 1.53 & 0.82 & 1.53 & 0.40 & 16.04 & 54.52 \\
\hline S8 & Yunnan & 5.34 & 7.49 & 20.35 & 1.56 & 1.53 & 0.84 & 1.52 & 0.37 & 15.64 & 54.64 \\
\hline S9 & Hebei & 2.95 & 2.53 & 12.97 & 0.96 & 0.96 & 0.31 & 1.26 & 0.21 & 8.87 & 31.02 \\
\hline S10 & Hebei & 2.82 & 2.84 & 13.00 & 0.99 & 0.81 & 0.31 & 1.27 & 0.22 & 8.76 & 31.02 \\
\hline S11 & Hebei & 2.93 & 2.96 & 13.06 & 0.95 & 0.84 & 0.33 & 1.31 & 0.24 & 8.90 & 31.52 \\
\hline S12 & Hebei & 2.88 & 3.07 & 13.65 & 1.07 & 0.84 & 0.34 & 1.25 & 0.22 & 9.23 & 32.56 \\
\hline S13 & Hebei & 3.05 & 3.17 & 12.86 & 1.10 & 0.89 & 0.33 & 1.27 & 0.23 & 9.36 & 32.26 \\
\hline S14 & Hebei & 3.07 & 3.15 & 12.88 & 1.11 & 0.93 & 0.33 & 1.30 & 0.22 & 9.04 & 32.03 \\
\hline S15 & Hebei & 3.03 & 3.11 & 13.13 & 1.16 & 0.99 & 0.33 & 1.29 & 0.19 & 9.18 & 32.41 \\
\hline S16 & Hubei & 3.84 & 4.32 & 16.83 & 1.17 & 1.09 & 0.47 & 1.05 & 0.13 & 12.88 & 41.78 \\
\hline S17 & Hubei & 3.87 & 4.28 & 16.75 & 1.21 & 1.07 & 0.46 & 1.07 & 0.11 & 12.75 & 41.56 \\
\hline S18 & Hubei & 3.95 & 4.42 & 16.99 & 1.23 & 1.07 & 0.48 & 1.07 & 0.11 & 13.09 & 42.41 \\
\hline S19 & Hubei & 3.86 & 4.23 & 17.16 & 1.20 & 1.09 & 0.52 & 1.10 & 0.11 & 13.38 & 42.66 \\
\hline S20 & Hubei & 3.94 & 4.35 & 17.21 & 1.23 & 1.12 & 0.52 & 1.12 & 0.12 & 13.54 & 43.14 \\
\hline S21 & Hubei & 3.90 & 4.27 & 17.11 & 1.22 & 1.16 & 0.51 & 1.11 & 0.10 & 13.00 & 42.38 \\
\hline
\end{tabular}

${ }^{\mathrm{a}}$ Values represent means $\pm \mathrm{SD}, n=9$.

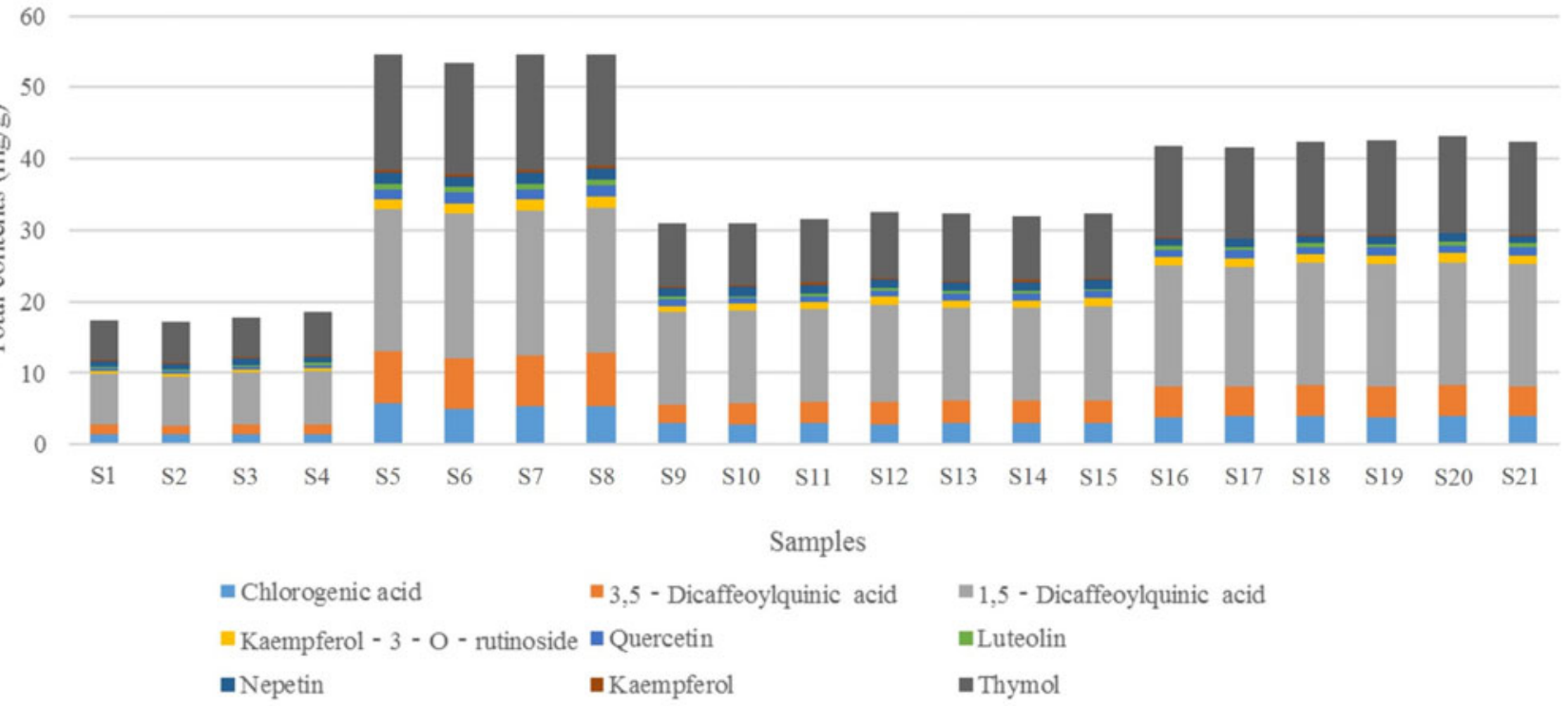

Figure 3. The total contents of 9 compounds in 21 samples.

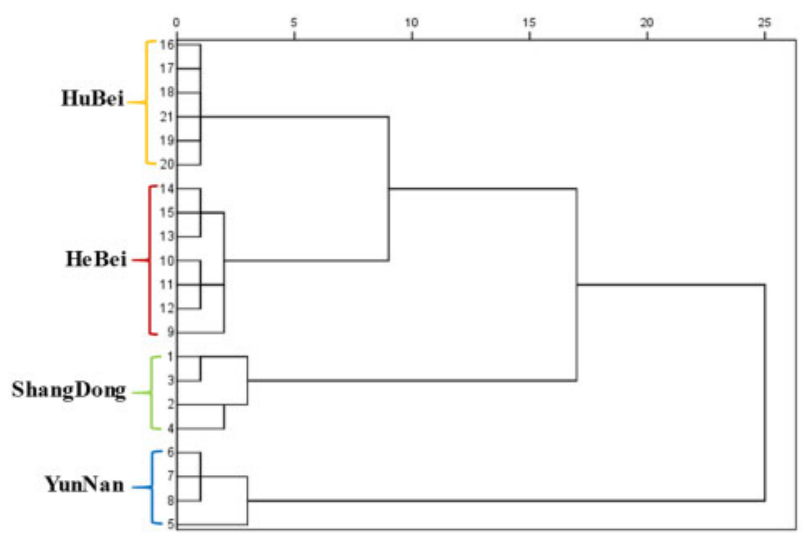

Figure 4. The diagram of cluster analysis of 21 batches of BINW samples.

including 10 flavonoids, 8 phenolic acids, and another 28 compounds, were tentatively identified. In addition, our results also confirmed that UHPLC-PAD as a rapid, accurate, and efficient method could be employed for qualitative analysis of 21 batches of BINW. Furthermore, our results also indicated that nine compounds could be marked as the quality control compounds for BINW. Together with quantitative data by the clusters analysis via PCA, our results show that a heatmap visualization could be used to analyze and discriminate the 21 batches of BINW from different areas through the quality control of 9 compounds. Lastly, our results also indicated that the Yunnan samples had the highest contents of the nine compounds (flavonoids, phenolic acids) and the best antioxidant activity among these samples from four different origins, which unambiguously indicated that the higher the total amount of flavones and phenolic 


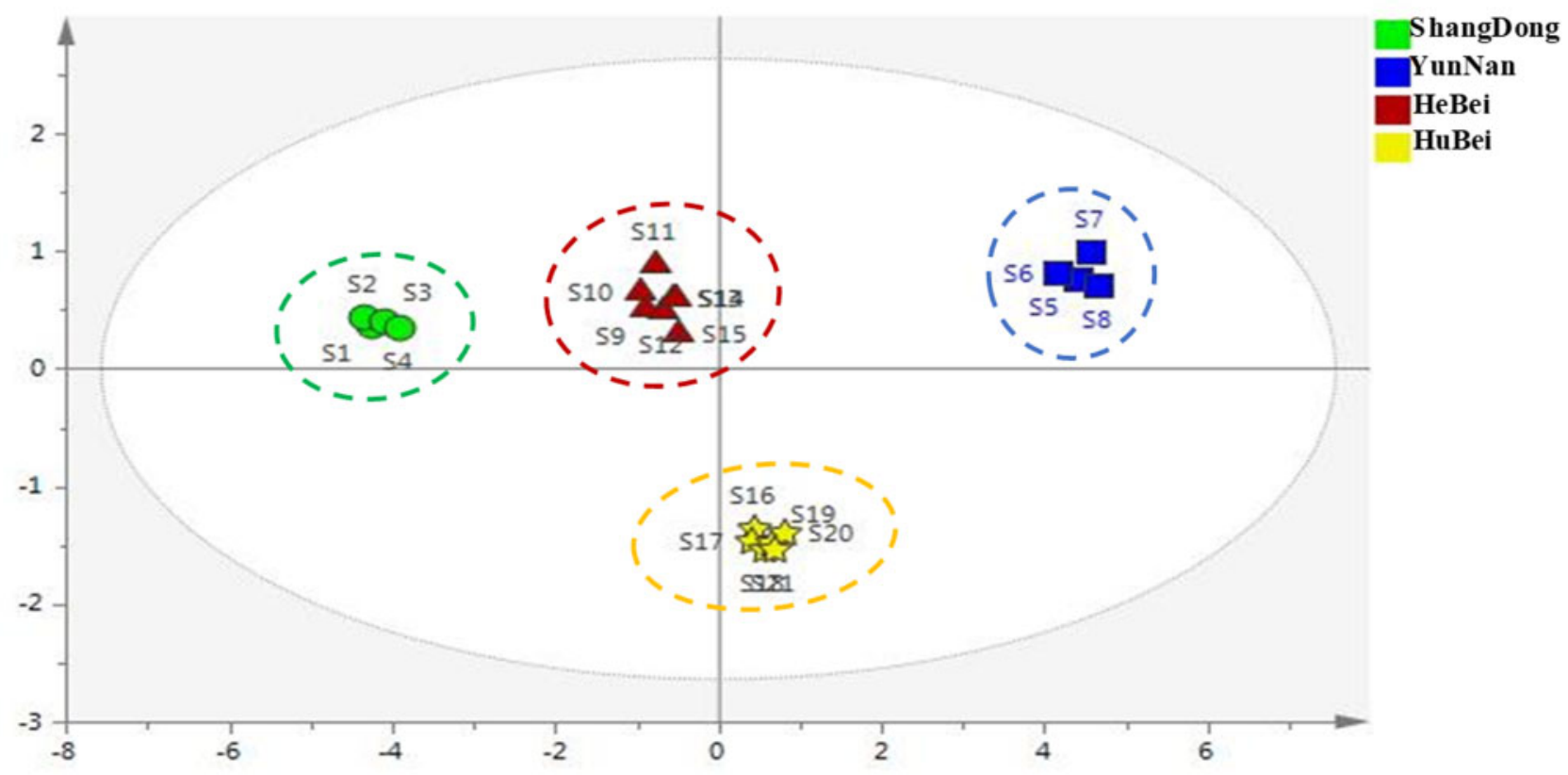

Figure 5. The PCA score plot of 21 batches of BINW samples based on the quantitative data of 9 compounds using Pareto scaling with mean centering. The green circle corresponds to Shandong province (S1-S4), blue square corresponds to Yunnan province (S5-S8), red triangle corresponds to Hebei province (S9-S15), and the yellow pentagram corresponds to Hubei province (S16-S21).

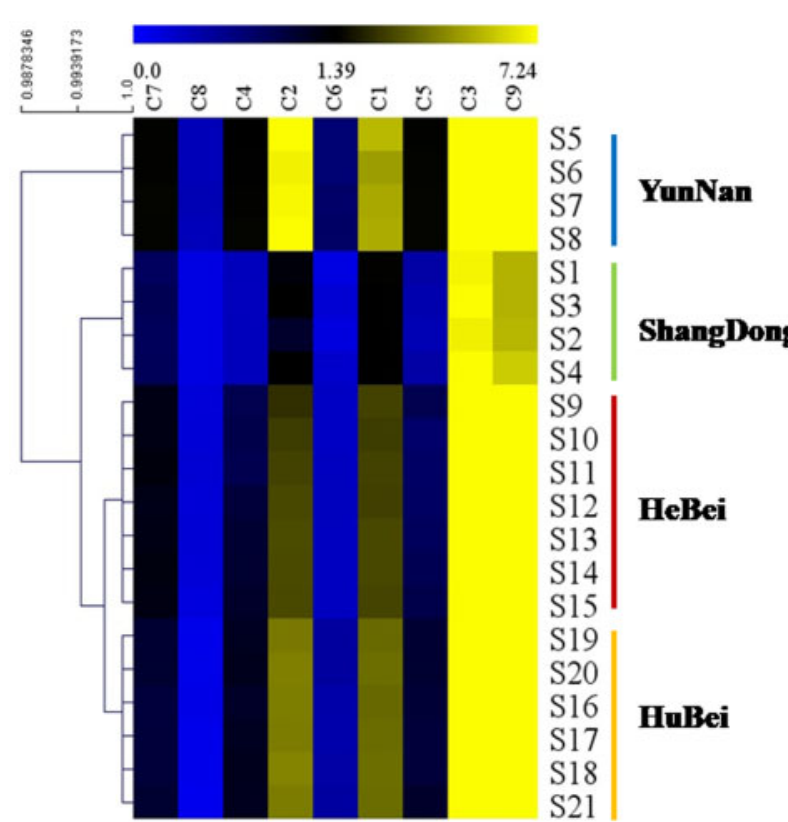

Figure 6. Hierarchical clustering analysis heatmap of nine compounds between Yunnan, Shandong, Hebei, and Hubei samples.

acids in the sample is, the better the antioxidant activity it shows. Taken together, our study not only developed a powerful platform to explain the different traditional Chinese medicines species that are closely related through chemometric and chemical profiling, but also presented a useful method to establish quality criteria of BINW with multiple origins. It is envisioned that the methods developed here could be extended to the many compound identifications and discriminations from other sources in the future.

\section{Acknowledgments}

This work was supported by Jiangxi Province Health and Family Planning Commission Chinese Medicine Research projects (No. 2018B144). We also acknowledge the funding support from National Key Research and Development Plan (No. 2017YFC1702906).

\section{Conflict of interest}

There is no conflict in this section.

\section{Supplemental information}

Supplemental information is available on the J. AOAC Int. website.

JAOAC does not publish color figures in the print version. Color images are published online only.

\section{References}

1. Caro, A.A., Davis, A., Fobare, S., Horan, N., Ryan, C., \& Schwab, C. (2019) Toxicol. Vitro. 54, 1-9. doi: 10.1016/j.tiv.2018.09.001

2. Turkan, F., Atalar, M.N., Aras, A., Gulcin, I., \& Bursal, E. (2020) Bioorg. Chem. 94, 103333.doi:10.1016/j.bioorg.2019.103333

3. Xiang, J., Apea-Bah, F.B., Ndolo, V.U., Katundu, M.C., \& Beta, T. (2019) Food Chem. 275, 361-368. doi:10.1016/j.foodchem. 2018.09.120

4. Wang, J., Gao, L., Lee, Y.M., Kalesh, K.A., Ong, Y.S., Lim, J., Jee, J.-E., Sun, H., Lee, S.S., Hua, Z.-C., \& Lin, Q. (2016) Pharmacol. Thera. 162, 10-22. doi:10.1016/j.pharmthera.2016.01.010

5. Xiao, Q., Zhu, W., Feng, W., Lee, S.S., Leung, A.W., Shen, J., Gao, L., \& Xu, C. (2019) Front. Pharmacol. 9, 1534.doi: 10.3389/fphar.2018.01534 
6. Franco, D., Munekata, P.E.S., Agregán, R., Bermúdez, R., López-Pedrouso, M., Pateiro, M., \& Lorenzo, J.M. (2020) Antioxidants 9, 90 doi:10.3390/antiox9020090

7. Chanhiguera, J.E., Santacruzortega, H., Carbonellbarrachina, A.A., Burgoshernandez, A., Roblessanchez, R.M., Cruzramirez, S.G., \& Ezquerrabrauer, J.M. (2019) Molecules 24, 3420.doi:10.3390/molecules 24193420

8. Mandal, A.K., Shakila, R., Thatipelli, S., Rubeena, M., Sunilkumar, K.N., Thilagam, A., \& Ganesan, R. (2019) Int. J. Pharm. Invest. 9, 128-134. doi:10.5530/ijpi.2019.3.24

9. Korkmaz, N., Sener, S.O., Akkaya, S., Badem, M., Aliyazicioglu, R., Abudayyak, M., Oztas, E., \& Ozgen, U. (2019) Turkish J Biochem. 44, 278-288. doi:10.1515/tjb-2017-0345

10. Wen, Z., Huang, W., Long, W., Gong, J., \& Wu, D. (2019) Biomed. Chromatogr. . 33, e4682.doi:10.1002/bmc.4682

11. Yan, L., Jin, H.-Z., Nie, L.-Y., Qin, J.-J., Fu, J.-J., \& Zhang, W.-D. (2011) Nat. Prod. Res. Dev. 23, 258-261. doi: 10.16333/j.1001-6880.2011.02.014

12. Yan, L., Cheng, X.R., Zeng, Q., Qin, J.J., Zhang, W.D., \& Jin, H.Z. (2011) Biochem. Syst. Ecol. 39, 700-703. doi:10.1016/ j.bse.2011.06.001

13. Yan, L., Huang, Y., Fu, J.-J., Qin, J.-J., Zeng, Q., Zhu, Y., Yan, S.K., Zhang, W.-D., \& Jin, H.-Z. (2010) HCA 93, 1418-1421. doi: 10.1002/hlca.200900432

14. Anna, H.E., She, C., Zeng, J., \& Peng, S. (2016) Chin. Pharm. Bull. 1, 79-83. doi:10.3969/j.issn.1001-1978.2016.01.017

15. Liu, P., Yang, G., Tian, X., Zhang, Y., Li, F., \& Wang, F. (2011) Sci. Tech. Food Ind. 10, 187-189. doi:10.13386/j. issn1002-0306.2011.10.026

16. Zhang, Y., Wang, F., \& Liu, P. (2011) Sci. Tech. Food Ind. 12, 441-443 + 446. doi:10.13386/j. issn1002-0306.2011.12.016

17. Zhang, Y.G., Liu, P.H., \& Wang, F. (2012) Sci. Tech. Food Ind. 21, 353-355 + 360. doi:10.13386/j. issn1002-0306.2012.21.016

18. Guanghong, Y., Yaguan, Z., Fan, W., \& Pinhua, L. (2011) J. Anhui Agric. Sci. 31, 19648-19649. doi:10.13989/j.cnki.05176611.2011.31.158

19. Lin, C., Lin, X., Lin, L., Wang, J., Lin, Z., \& Lin, J. (2015) Chin. Chem. Lett. 26, 1225-1230. doi:10.1016/j.cclet.2015.03.007

20. Dai, L., Guo, N., Liu, Y., Shen, S., Ge, Q., \& Pan, Y. (2019) Chin. Chem. Lett. 30, 103-106. doi:106. 10.1016/j.cclet.2017.12.023

21. Liu, Y., Xun, X., Yi, J., Xiang, Y., \& Hua, J. (2017) Chin. Chem. Lett. 28, 1093-1098. doi:10.1016/j.cclet.2016.11.026

22. Sun, C., He, J., Wu, H., Song, X., Li, J., Huang, L., Xu, X., Wang, M., Zhang, R., \& Abliz, Z. (2018) Chin. Chem. Lett. 29, 1284-1286. doi:10.1016/j.cclet.2018.01.015

23. Cong, Y., Guo, J., Tang, Z., Zhang, Q., \& Cai, Z. (2014) Chin. Chem. Lett. 25, 1107-1111. doi:10.1016/j.cclet.2014.05.016

24. Wang, Z., Wang, Q., Han, Y., Jiang, M., Gao, J., Miao, Y., \& Bai, G. (2017) Chin. Chem. Lett. 28, 476-481. doi:10.1016/j.cclet.2016.11.021

25. Lin, Z., \& Pan, L. (2008) J. Guangdong Coll. Pharm. 1, 9-11. doi: 10.16809/j.cnki.1006-8783.2008.01.005

26. Ke, L.I., Lei-zhe, S.H.I., Dan, C., Hui-bing, H.U., Mao-fang, L.U., Ruo-cun, L.I., \& Shun-xiang, L.I. (2013) J. Trad. Chin. Med. Univ. Hunan 33, 41-44. doi:10.3969/j.issn.1674-070X.2013.07.010.041.04

27. Loizzo, M.R., Pugliese, A., Bonesi, M., Tenuta, M.C., Menichini, F., Xiao, J., \& Tundis, R. (2016) J. Agric. Food Chem. 64, 2467-2474. doi:10.1021/acs.jafc.5b03092

28. Benzie, I.F., \& Strain, J. (1996) J. Anal. Biochem. 239, 70-76. doi: 10.1006/abio.1996.0292

29. Mikulic-Petkovsek, M., Samoticha, J., Eler, K., Stampar, F., \& Veberic, R. (2015) J. Agric. Food Chem. 63, 1477-1487. doi: 10.1021/jf506005b
30. Zhang, L., Xu, L., Ye, Y-H., Zhu, M-F., Li, J., Tu, Z.-C., Yang, SH., \& Liao, H. (2019) Ind. Crop. Prod. 129, 156-168. doi: 10.1016/j.indcrop.2018.11.051

31. Putri, R.A., Safni, S., Jamarun, N., Septiani, U., Kim, M., \& Zoh, K. (2019) Egypt. J. Chem. 63, 563-575. doi: 10.21608/ejchem.2019.14543.1883

32. Fan, W., Fan, L., Peng, C., Zhang, Q., Wang, L., Li, L., Wang, J., Zhang, D., Peng, W., \& Wu, C. (2019) Molecules 24, 359.doi: 10.3390/molecules24020359

33. Zhang, Y., Shi, J., Ni, Y., Liu, Y., Zhao, Z., Zhao, X., \& Gao, Z. (2019) Sci. Rep. 9, 1-14. doi:10.1038/s41598-019-54836-1

34. Zhang, M., Liu, H.J., Huang, X.L., Shao, L.J., Xie, X.L., Wang, F., Yang, J., Pei, P., Zhang, Z.Q., Zhai, Y.C., Wang, Q., Zhang, T., Huang, J., \& Cui, X.D. (2019) J. Chromatogr B. 1112, 33-40. doi: 10.1016/j.jchromb.2019.02.028

35. Zhu, D., Kebede, B.T., Chen, G., Mccomb, K., \& Frew, R.D. (2020) Food Control 116, 107017 doi:107017. 10.1016/j.foodcont. 2019.107017

36. Zhang, L., Li, Y., Liang, Y., Liang, K., Zhang, F., Xu, T., Wang, M., Song, H., Liu, X., \& Lu, B. (2019) Food Chem. 276, 538-546. doi:10.1016/j.foodchem.2018.10.074

37. Bursal, E., Aras, A., \& Kllıç, Ö. (2019) Nat. Prod. Res. 33, 1975-1979. doi:10.1080/14786419.2018.1480018

38. Sun, S., Liu, M., He, J., Li, K., Zhang, X., \& Yin, G. (2019) Molecules 24, 1791.doi:10.3390/molecules24091791

39. Huang, W., Tan, T., Li, Z., Ouyang, H., Xu, X., Zhou, B., \& Feng, Y. (2018) J. Pharm. Biomed. Anal. 154, 236-244. doi: 10.1016/j.jpba.2018.02.020

40. Huang, W., Wen, Z., Wang, M., Xu, B., Zhou, B., \& Li, X. (2020) Biomed Chromatogr. 34, e4736.doi:10.1002/bmc.4736

41. Grauso, L., Emrick, S., De Falco, B., Lanzotti, V., \& Bonanomi, G. (2019) Phytochem. Rev. 18, 1115-1132. doi:10.1007/s11101019-09622-2

42. Mackled, M.I., EL-Hefny, M., Bin-Jumah, M., Wahba, T.F., \& Allam, A.A. (2019) Processes. 7, 861.doi:10.3390/pr7110861

43. Herz, W., \& högenauer, G. (1961) J. Org. Chem. 26, 5011-5013. doi:10.1021/jo01070a056

44. Haq, F.U., Ali, A., Akhtar, N., Aziz, N., Khan, M.N., Ahmad, M., \& Musharraf, S.G. (2020) J Adv. Res. 24, 79-90. doi: 10.1016/j.jare.2020.02.001

45. Shu-Jun, Z., Huan, L., Jun, L, Jin-Lan, W., \& Wen-Zhi, Z. (2015) Chin. Trad. Herbal Drugs 46, 329-333. doi:10.7501/j.issn.02532670.2015.03.004

46. Li, W., Feng, Y., Li, T., Wu, H., Li, Y., Zhong, G., Wu, B., \& He, M. (2016) J. Chin. Mass Spectr. Soc. 37, 504-516. doi: 10.7538/zpxb.youxian.2016.0028

47. Ouyang, H., Li, J., Wu, B., Zhang, X., Li, Y., Yang, S., He, M., \& Feng, Y. (2017) J. Chromatogr A. 1502, 38-50. doi:org/10.1016/ j.chroma.2017.04.05

48. Fu, Q., Tong, C., Guo, Y., Xu, J., Shi, F., Shi, S., \& Xiao, Y. (2020) Anal. Bioanal. Chem. 412, 321-333. doi:10.1007/s00216019-02238-7

49. Zhu, L.-L., Fan, L.-D., Hu, M.-H., MA, F.-L., \& Qi, J. (2019) Chin. J. Nat. Med. 17, 535-544. doi:10.1016/S1875-5364(19)30075-5

50. Yan, L. (2010) Shanghai Jiaotong University

51. Ambrož, M., Šmatová, M., Šadibolová, M., Pospíšilová, E., Hadravská, P., Kašparová, M., Skarková, V.H., Králová, V., \& Skálová, L. (2019) Acta Pharm. 69, 121-128. doi:10.2478/ acph-2019-0003

52. Liu, C. (2012) Graduation thesis, Shanghai Jiaotong University

53. Zeng, S., Ji, D., \& Liu, H. (2015) Flavour. Fragrance Cosmetics 4, 77-82 